\title{
CHROMOSOME PAIRING AND CHIASMA FORMATION IN WHEAT PLANTS TRIISOSOMIC FOR THE LONG ARM OF CHROMOSOME 5B
}

\author{
by
}

\author{
BENTE WISCHMANN
}

Department of Physiology, Carlsberg Laboratory

Gamle Carlsberg Vej 10, DK-2500 Copenhagen Valby

Keywords: Electron microscopy, synaptonemal complex, recombination nodule, dosis effect, Triticum aestivum

\begin{abstract}
Chromosome pairing and chiasma formation have been analyzed at the light and electron microscopic levels in wheat plants triisosomic for the long arm of chromosome 5B. Complete reconstruction of the chromosome complement from one zygotene nucleus, as well as analyses of several hundred spread and silver stained nuclei, showed that pairing was arrested at the beginning of zygotene, the mean degree of pairing in five nuclei being 25 percent (range 8-37). At this stage, multiple associations of chromosomes were present, signifying pairing both between partly-homologous and nonhomologous chromosome segments. A few multivalents were present at metaphase I together with bivalents and univalents. The chiasma frequency (a mean of 18 per cell) was only half of that seen in normal wheat. In spread nuclei at late diplotene, a mean of 41 segments of synaptonemal complex was counted, 20 of which had thickened lateral components. The results are discussed in relation to the possible dosage effect of the $\mathrm{Ph}$ gene located on the long arm of chromosome 5B.
\end{abstract}

\section{INTRODUCTION}

The disomic inheritance observed in allohexaploid wheat Triticum aestivum $(2 \mathrm{n}=$ $14 \mathrm{~A}+14 \mathrm{D}+14 \mathrm{~B}=42$ ) is controlled by a number of genes on different chromosome arms, the most important one being the $\mathrm{Ph}$ gene (or genes) located an the long arm of chromosome $5 B$ (5BL) (23). When two copies of this gene are present, as in euploid wheat, crossing over and chiasma formation occur only between homologous chromosomes (i.e., between chromosomes belonging to the same genome), and only bivalents are observed at metaphase I. If both 5B chromosomes are lacking $(2 n=14 A+14 D+$ $12 \mathrm{~B}=40$ ), crossing over is no longer restricted to homologues but can also occur between the so-called homoeologous chromosomes i.e., partly homologous chromosomes from the different genomes. This leads to the formation of multivalents and to translocations between homoeologous chromosomes (22).

Several hypotheses have been presented to explain this genetic control (23). In a series of investigations FELDMAN et al. $(3,4,10,28)$ suggested that the $\mathrm{Ph}$ gene controls the location of chromosomes and genomes in the interphase nucleus. With two doses of the gene, a close alignment of homologues effectively prevents interlocking of chromosomes and pairing between homoeologues during zygotene. In the absence of this gene, homologous and homoeologous chromosomes are associated prior to zygotene, and consequently pairing and interlocking of homoeologous chromosomes may occur.

This hypothesis has been deduced from metaphase plates of root tip cell squashes studied under the light microscope. In such prepara- 
tions, cytologically marked homologues (telocentric chromosomes) lie closer together than homoeologues. It has been assumed that this is also the case for the normal unmarked homologues and further that the relative spatial distribution at premeiotic interphase is the same as at metaphase in somatic tissue $(3,4)$. Recent ultrastructural analyses of nuclei of euploid wheat have, however, revealed chromosome interlocking as well as multivalents at zygotene (12, 16).

Multivalents at zygotene have also been reported in other polyploid species, e.g., Bombyx, Lolium $(17,18,20)$. In the absence of crossing over, most multiple associations transform into bivalents during zygotene or early pachytene by dissolution and reformation of the central region of the synaptonemal complex. Furthermore, interlocking of chromosomes frequently occurs during zygotene pairing in diploid as well as in polyploid species (see 27 for review). In most cases there are no interlocking bivalents at pachytene and it has been shown $(13,19)$ that the interlocked chromosomes are released during zygotene and early pachytene by breakage and repair of the chromosomes involved. These observations conflict with the notion that premeiotic alignment of homologues is the sole determinant of exclusive bivalent formation and the absence of interlocking observed at metaphase I.

Light microscopical studies by FELDMAN (10) of metaphase I chromosomes of wheat plants with four doses of $5 \mathrm{BL}$ in the form of two isochromosomes $\quad(2 \mathrm{n}=14 \mathrm{~A}+14 \mathrm{D}+12 \mathrm{~B}+2$ iso$5 B L=42$ ) revealed a slight reduction in the chiasma frequency and only bivalents at metaphase I, Six doses of $5 B L(2 n=14 A+14 D+12 B+3$ iso$5 \mathrm{BL}=43$ ) caused a 50 percent reduction of the chiasma frequency and the failure of regular bivalent formation as evidenced by the presence of univalents, a few multivalents and heteromorphic bivalents at metaphase I. The dosage of 5BL also affects the frequency of interlockings observed at metaphase I, the frequencies being low in euploid wheat while the presence of 0,4 and 6 copies of SBL is accompanied by a stepwise increase of the frequency.

FELDMAN (10) explained the dosage effect of $5 \mathrm{BL}$ in the following way: In the absence of $5 \mathrm{BL}$, homologous and homoeologous chromosomes are so closely associated at premeiotic interphase that the formation of multivalents and interlocking occurs during zygotene. When six doses of $5 \mathrm{BL}$ are present, all premeiotic chromosome associations are suppressed with distortion of zygotene pairing and consequent interlocking.

From the normal course of meiosis in other polyploid species, НовOLTH (12) speculated that the increase in copy number of 5BL affected the time of crossing over relative to the transformation of multivalents into bivalents. In the absence of 5BL, crossing over takes place before multivalents are corrected, while six doses of 5BL postpone crossing over to a period no longer optimal for reciprocal recombination. This proposal implies that the formation of interlockings is independent of the gene dosage. The retention of interlockings at metaphase I in plants with 0 , 4 and 6 doses of 5BL is also unexplained.

The present work describes meiotic prophase in wheat carrying three isochromosomes of $5 \mathrm{BL}$. It is based on serial sectioning and spreading of meiotic nuclei, the former technique being superior with respect to ultrastructural detail while quantitative information is more easily obtained with the latter.

\section{MATERIALS AND METHODS}

\subsection{Materials}

As described by FELDMAN (10) wheat plants containing three isochromosomes for the long arm of chromosome 5B (triisosomic 5BL) can be obtained by selfing plants with two isochromosomes for this chromosome arm. Seeds of Triticum aestivum cv. Chinese Spring plants diisosomic for the long arm of chromosome $5 \mathrm{~B}$ (diisosomic 5BL), were kindly provided by Dr. E.R. SEARS, University of Missouri, and propagated in the Royal College of Forestry phytotron in Stockholm. Three lots of 800 seeds were grown in a green house at $20{ }^{\circ} \mathrm{C}$ under continuous light.

Triisosomic 5BL plants were identified by phenotypic characteristics and by orcein squashes of metaphase I cells as described in section 3.1 . 


\subsection{Methods}

\subsection{1. $N$-banding}

The procedure for $\mathrm{N}$-banding was a modification of that recommended by ENDO and GILL (9). Seeds from diisosomic 5BL plants were allowed to germinate for 36 hours at room temperature, and the root tips were excised and immersed in icewater for 22 hours. The root tips were subsequently fixed in a 3:1 mixture of absolute ethanol and glacial acetic acid for at least 3 hours at room temperature. They were then treated with a cellulase-pectinase solution ( $1 \%$ cellulase, 4104 , Institute of Biochemistry, Shanghai, Academia, Sinica, and $1 \%$ pectinase, 4443-01, Koch - Light Laboratories Ltd.) for 2 hours at $30^{\circ} \mathrm{C}$ and refixed in the $3: 1$ absolute ethanol glacial acetic acid mixture for 3 hours at $4{ }^{\circ} \mathrm{C}$. The root tips were then squashed in $45 \%$ acetic acid and N-banded according to the procedure of ENDO and GILL (9).

\subsubsection{Electron microscopy}

According to JENKINS (16) only about $20 \%$ of the microsporocytes in meiotic prophase are adequately preserved following conventional fixation for electron microscopy. A significant improvement (fourfold) was observed, when the spikes were fixed in vacuo. This improvement could, however, not be obtained with the present material and a procedure was developed where the spikes were fixed under high pressure.

Spikes at the desired stage, as judged by their length, were excised from the triisosomic 5BL plants and placed in a small glass tube containing a $4 \%$ solution of glutaraldehyde (purification index, $A_{235} / A_{280}$ of 0.1 , ANDERSON (1)) in 0.06 M-Na-phosphate buffer (pH 7.4). The glass tube was placed inside a Yeda press connected to a $\mathrm{N}_{2}$-flask. The outlet of the Yeda press was equipped with a needle valve leak and a gas flowmeter. The start pressure was 100 bar and the leak was adjusted so that the pressure declined to 50 bar in about 2 hours. Next morning when the system had reached or almost reached atmospheric pressure the spikes were taken out, washed and stored in phosphate buffer at $4{ }^{\circ} \mathrm{C}$ until the next day. About $90 \%$ of the anthers fixed in this way were useful for electron microscopy.
The anthers, preferentially the ones from the first floret, were dissected out and their length measured. Anthers ranging in length from 0.9 $\mathrm{mm}$ to $1.3 \mathrm{~mm}$ contained microsporocytes at stages from leptotene to metaphase I. A few anthers were squashed in phosphate buffer and viewed under phase contrast optics to check the quality of the fixation. One half of the remaining anthers were stained in a $1 \%$ phophotungstic acid (PTA) solution in absolute ethanol at $4{ }^{\circ} \mathrm{C}$ overnight and the other half postfixed in $\mathrm{OsO}_{4}$ (16). Following dehydration in an ethanol series the anthers were embedded in Spurr's low viscosity resin and polymerized overnight at $70^{\circ} \mathrm{C}$. Thick survey sections (approximately $2 \mu \mathrm{m}$ in thickness) of the embedded anthers were examined under the light microscope for a preliminary determination of meiotic stage. Thin sections ( $80 \mathrm{~nm}$ in thickness) of nuclei at the desired stage were cut on a Reichert Om U3 ultramicrotome equipped with a diamond knife. A few thin sections were analysed for a precise stage determination before serial sections were cut. The $\mathrm{OsO}_{4}$ fixed material was double stained in uranyl acetate and lead citrate in a LKB UltroStainer 2168 , System Carlsberg. The serial sections were examined in a Siemens 102 electron microscope at $80 \mathrm{kV}$, photographed at a magnification of 3,000 times and printed to a final magnification of 17,100 or 18,500 times. The three dimensional reconstructions of the synaptonemal complexes and the lateral components, as well as the length measurements were performed as described by RASMUSSEN and HOLM (19).

In the present study three nuclei, two PTA stained and one $\mathrm{OsO}_{4}$ fixed, were serially sectioned, photographed and printed. One PTA stained nucleus was completely reconstructed.

\subsubsection{Spreading}

The procedure used for isolating and spreading meiotic nuclei of wheat was developed by P.B. HOLM and can be described as follows. Anthers are macerated in a medium containing $0.5 \% \mathrm{NaCl}, 10 \mathrm{mM}$-EDTA and $6 \mathrm{mM}-\mathrm{Na}$-phosphate buffer, $\mathrm{pH}$ 7.5. Swelling of the nuclei is induced by addition of a few drops of maceration medium containing $0.03 \%$ detergent (Tex-I, Blumøller, DK). Following fixation with a $4 \%$ 


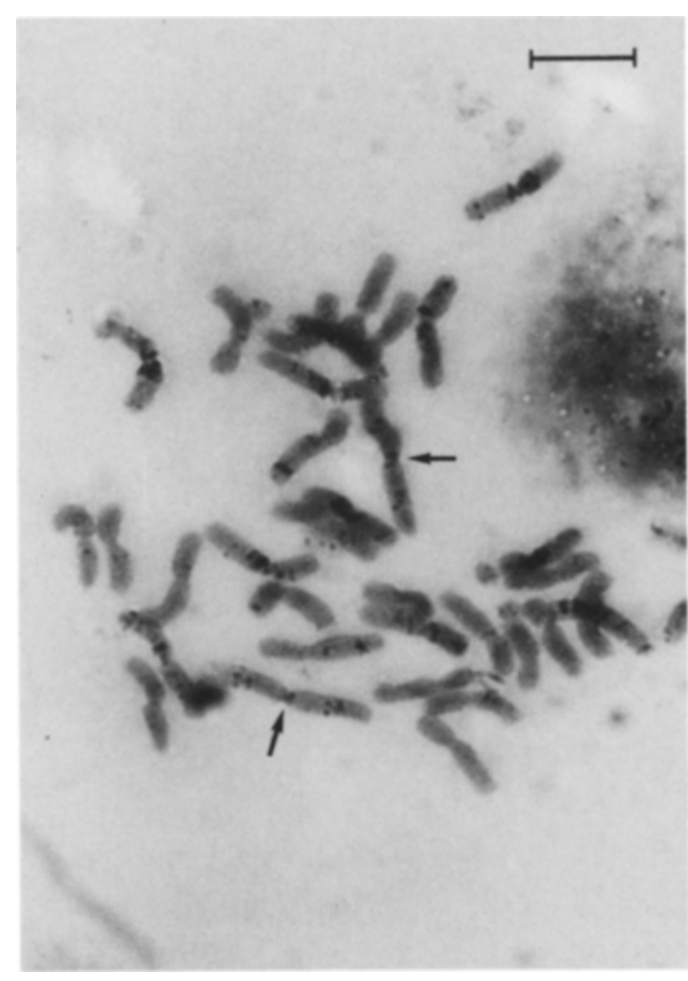

Figure 1. A photograph of an $\mathrm{N}$-banded mitotic metaphase from root tips of germinated seeds diisosomic for 5BL. The two isochromosomes are shown and the positions of their centromeres are denoted by arrows. $(\mathrm{Bar}=10 \mu \mathrm{m})$. formaldehyde solution containing $1.5 \%$ sucrose, $\mathrm{pH} \mathrm{8.2,} \mathrm{the} \mathrm{suspension} \mathrm{of} \mathrm{nuclei} \mathrm{is} \mathrm{poured} \mathrm{onto}$ slides covered with an Optilux membrane and allowed to air-dry overnight. After washing, the spread nuclei were stained with $\mathrm{AgNO}_{3}$ as described by GOODPASTURE and BLOOM (11) and Bloom and GoOdPasture (6).

The spread and stained nuclei were photographed in a Siemens 102 electron microscope at $80 \mathrm{kV}$ at a magnification of 3,000 times. The negatives were printed to a final magnification of 14,900 times and montages were made from these prints. The length of the lateral components was measured on the montages with a map measurer.

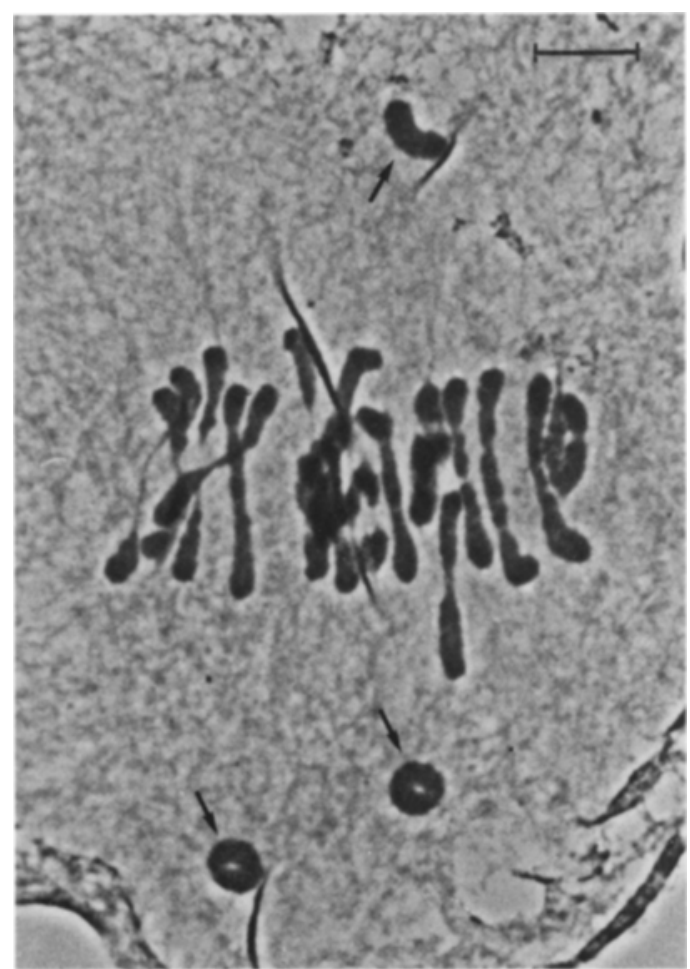

Figure 2. A photograph of an orcein stained meiotic metaphase I from pollen mother cells of plants triisosomic for 5BL. The three isochromosomes (two rings and one rod) are denoted by arrows. (Bar $=10 \mu \mathrm{m}$ ).

\section{RESULTS}

\subsection{Identification of diisosomic 5BL plants}

Plants with two isochromosomes for the long arm of chromosome 5B are not phenotypically different from normal euploid wheat. Usually their chromosomal constitution is determined by light microscopic analysis of squashed metaphase I cells, where the two isochromosomes generally can be identified as small, univalent rings, the result of a chiasma between the identical arms. To verify that the isochromosomes consisted of the long arms of chromosome 5B, $\mathrm{N}$-banding of root tip metaphase chromosomes was performed.

As illustrated in Figure 1 mitotic metaphases contain two isobrachial chromosomes with perfectly symmetrical banding patterns, i.e., a faint proximal band and a more prominent double 


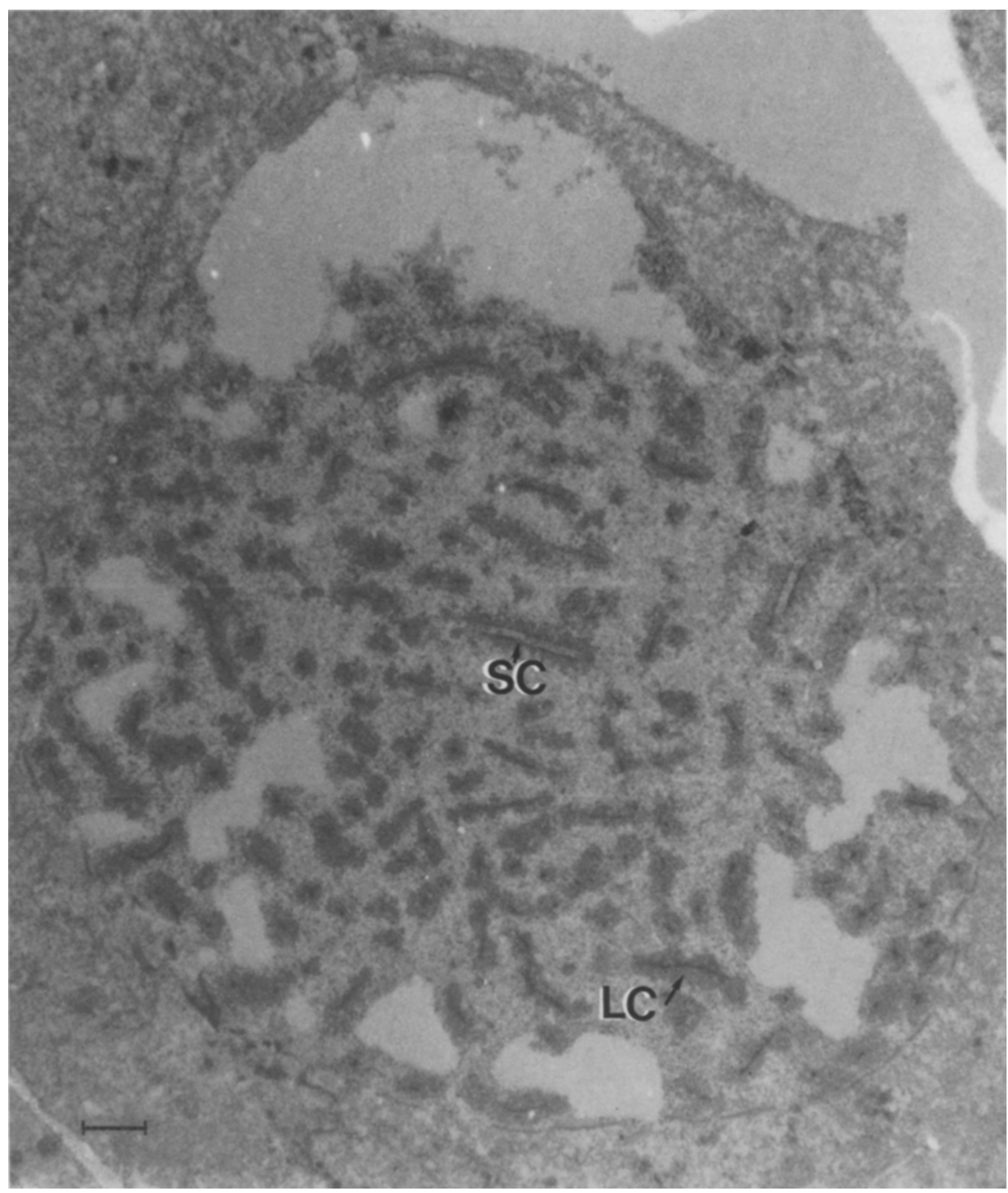

Figure 3. Survey micrograph of a PTA stained zygotene nucleus showing stretches of unpaired lateral components (LC) and synaptonemal complexes (SC). A large number of electron transparent cavities are seen in the nucleus. These are considered to be fixation artifacts. $(\mathrm{Bar}=1 \mu \mathrm{m})$.

band in the middle of the arm. This banding pattern is characteristic for the long arm of chromosome 5B (9) and shows that the chromosomes are true isochromosomes for the long arm of this chromosome.

\subsection{Identification of triisosomic 5BL plants} According to SEARS (personal communication) selfing of diisosomic 5BL plants gives rise to about $3 \%$ plants with three isochromosomes for the long arm of chromosome $5 \mathrm{~B}$. These can 


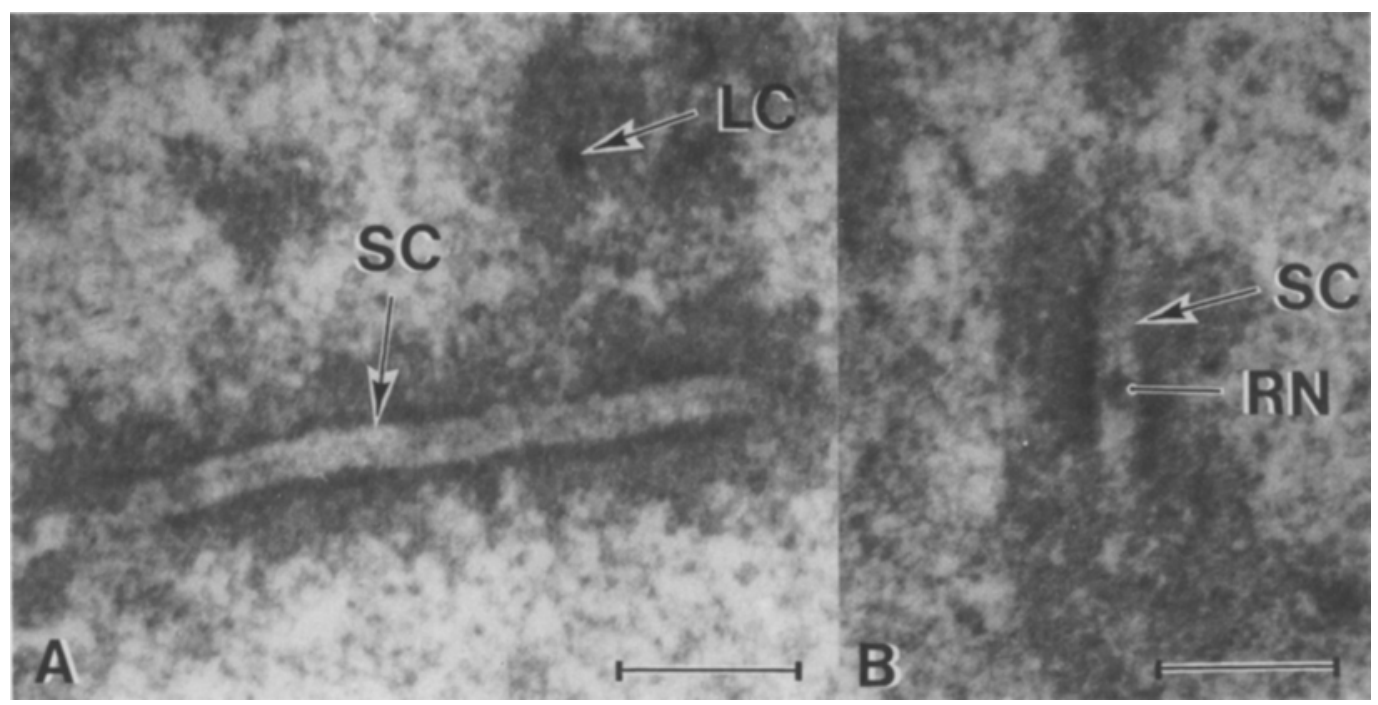

Figure 4. High magnification micrographs showing a synaptonemal complex (SC) and an unpaired lateral component (LC) (Figure 4A) and synaptonemal complex (SC) with a recombination nodule (RN)(Figure 4B). (Bar $=0.5 \mu \mathrm{m}$ ).

be recognized phenotypically as dwarfed plants.

The three lots of 800 diisosomic 5BL seeds gave rise to 27,27 and 20 dwarf plants, with two distinctly different phenotypes, one with normal broad leaves and one with narrow leaves. The plants with narrow leaves had empty loculi, while orcein squashes of pollen mother cells at metaphase I from dwarf plants with normal broad leaves revealed the presence of three isochromosomes. The isochromosomes were usually located outside the metaphase plate as univalent rings or rods (Figure 2). Univalents and rod bivalents were often observed whereas ring bivalents were rare. The mean number of chiasmata counted in 30 metaphases was $17.9 \pm$ 2.4. Interlocked bivalents and presumptive multivalents were frequently seen. Disjunction at anaphase I was highly irregular. The microsporocyte population in the individual anther was usually less synchronous than in euploid plants, the developmental stages ranging from zygotene to metaphase I - anaphase I.

\subsection{Three dimensional reconstruction}

\subsubsection{General aspects}

Two zygotene nuclei stained with PTA and one zygotene nucleus postfixed with $\mathrm{OsO}_{4}$ were serially sectioned and photographed. As illustrated in Figures 3 and 4 the lateral components can easily be distinguished from the chromatin after PTA staining, the difference in electron density being higher than that observed in material fixed and stained in the conventional way. One of the PTA stained nuclei was therefore selected for reconstruction. It was not possible to identify the centromeres after PTA staining in this material, in contrast to that reported by JENKINS (16). In the $\mathrm{OsO}_{4}$ fixed nucleus the centromeres appeared less electron dense than the flanking chromatin and the 43 centromeres ( 40 chromosomes +3 isochromosomes) were easily identified. The fine structure of the unpaired lateral components and the synaptonemal complexes as well as their dimensions were identical to that reported for normal wheat $(12,16)$.

In the completely reconstructed nucleus, 75 of the 86 telomeres were attached to the inner membrane of the nuclear envelope, the others were free in the nucleoplasm. The majority of the attachment sites were confined to a limited area of the nuclear envelope (Figure 5).

A single nucleolus was present, located adjacent to the inner nuclear membrane. Five un- 


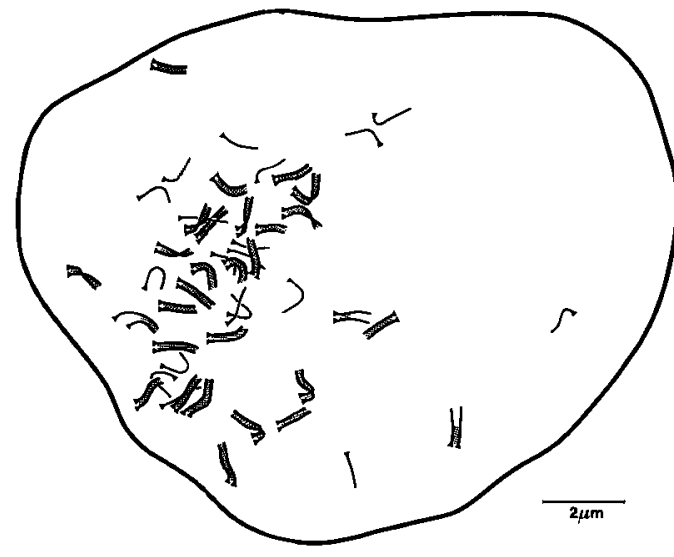

Figure 5. A reconstruction showing the distribution of the telomere attachment sites on the inner membrane of the nuclear envelope. paired chromosome segments entered the nucleolus through spherical regions of low electron density, the nucleolus organizing regions. One of the chromosome segments belonged to a pentavalent association (number 42 in Figure 9), one to a trivalent association (number 61 in Figure 9 and Figure 7) while the remaining three were segments not involved in multivalent associations. The lateral components were all interrupted in the nucleolus organizing regions and it was thus not possible to identify the remainder of the nucleolus organizing chromosomes. Light microscopical studies using in situ hybridization with labeled rRNA have identified major nu-

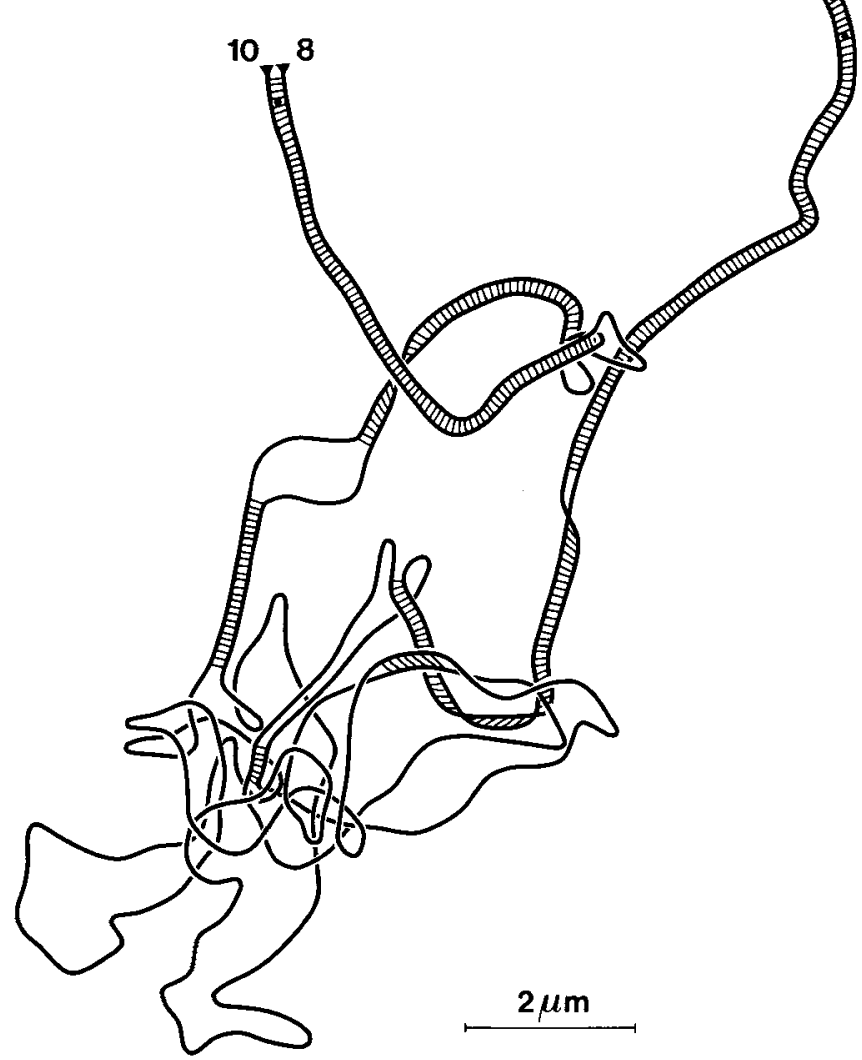

Figure 6. A reconstruction of a partially paired bivalent. Unpaired lateral components are represented by single lines, synaptonemal complexes by parallel lines with cross hatching. The recombination nodules are denoted by small circles. The telomeres are attached to the nuclear envelope and are represented by filled triangles. The numbers 8 and 10 relate to the length of the lateral components, cf. Figure 9. 


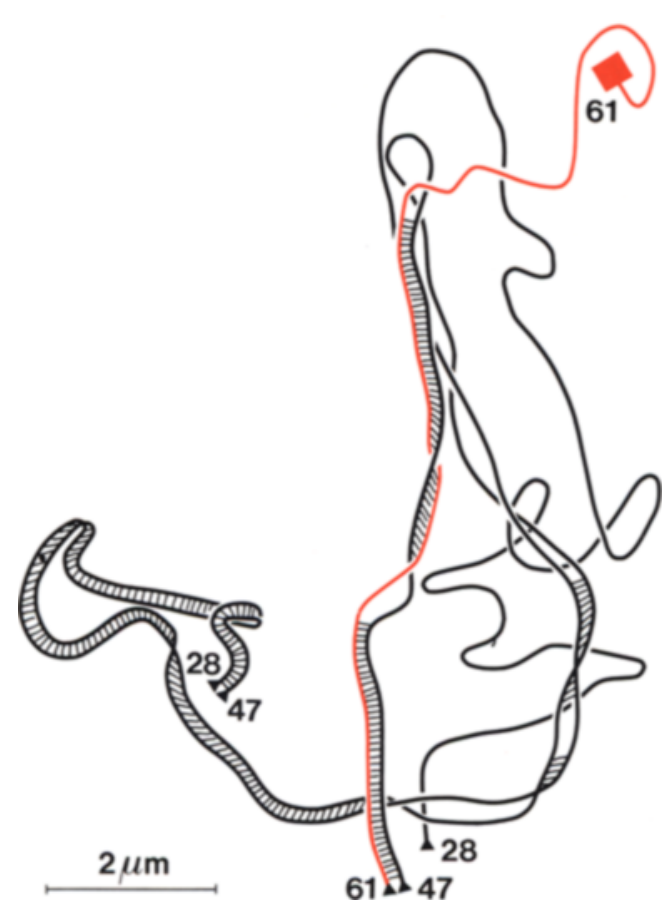

Figure 7. A reconstruction of a trivalent. The black square represents a nucleolus organizer region. The other symbols are described in the legend to Figure 6 . The numbers 28,47 and 61 relate to the length of the lateral components, cf. Figure 9.

cleolus organizing regions on chromosomes $1 \mathrm{~B}$ and $6 \mathrm{~B}$, and a minor organizing region on chromosome 5D while chromosome 1A, earlier assumed to possess ribosomal cistrons, appears to lack nucleolus organizing capacity (2). The present result suggests that not all the six potential organizing regions were active in the reconstructed nucleus.

\subsubsection{Chromosome pairing}

The complement of unpaired lateral components and synaptonemal complexes of the reconstructed nucleus is diagrammed in Figure 9 and reconstructions of different pairing configurations are shown in Figures 6,7 and 8. The total length of the lateral components amounted to $3,443 \mu \mathrm{m}$. Twenty-five percent of the complement was paired with a synaptonemal complex.
As shown in Figure 9 long continuous segments of synaptonemal complex were almost exclusively seen in the distal regions, 58 of the telomeres being paired with a synaptonemal complex. In addition, several interstitial sites for initiation of synaptonemal complex formation were found.

The complete reconstruction of the chromosome complement revealed several pairing configurations where more than two chromosomes were involved. As seen in Figure 9, the nucleus contained one association involving 9 chromosomes, one association of 5 chromosomes, six of 3 chromosomes and twelve associations of 2 chromosomes. In addition, 27 segments of unpaired lateral components were found. In Figure 9 lateral components are numbered according to decreasing length. In the 8 multiple associations each chromosome never exchanges pairing partner more than once.

Fifteen lateral components could be traced from one telomere to the other. The rest either ended free in the nucleoplasm (denoted by a cross in Figure 9), or entered the nucleolus (denoted by a black square in Figure 9) or a knot (denoted by a stippled line in Figure 9).

Only one of the twelve configurations involving two chromosomes could be traced from telomere to telomere. A reconstruction of this bivalent is shown in Figure 6, the lengths of the lateral components (numbered 8 and 10), differing by about $4 \%$. A larger difference in length of interstitially unpaired lateral components was found in one of the associations of three chromosomes, where the unpaired interstitial segments of the lateral component number 55 is about $25 \%$ longer than the corresponding segment of number 58 (Figure 9). In the remainder of the pairing configurations interstitial unpaired segments were always of the same length.

The reconstructed nucleus contained one example of a possible nonhomologous pairing, where a short segment of synaptonemal complex combines two unpaired lateral components of opposite polarity (in Figure 8). It was not possible to identify any of the paired and unpaired chromosomes and it is thus not known whether the different chromosome associations consist of homologous, homoeologous or nonhomologous chromosomes. It is likely, however, that most if not all of the associations of two chromo- 


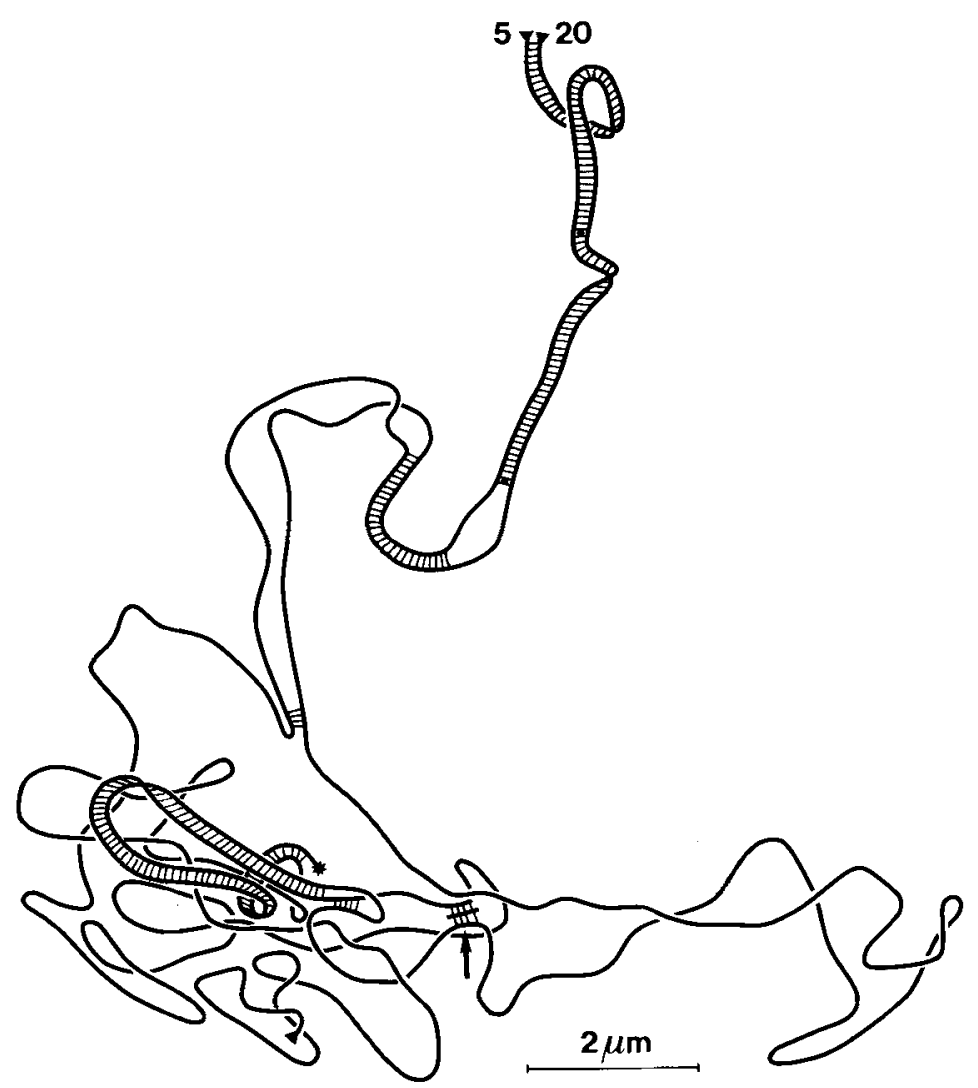

Figure 8. A reconstruction of a partially paired bivalent. One of the lateral components ends free in the nucleoplasm (denoted by an asterisk). Short segments of the two lateral components are paired with a small polycomplex consisting of two central regions associated by a lateral component-like structure (denoted by arrow). To the left of this region two seemingly antiparallel segments are paired with a synaptonemal complex. The other symbols are described in the legend to Figure 6 . The numbers 5 and 20 relate to the length of the lateral components, cf. Figure 9.

somes represent homologously paired chromosomes. Associations of three chromosomes probably consist of two homologues and one homoeologue and may thus be regarded as trivalents.

The association of nine chromosomes may be interpreted as consisting of two quadrivalents of homologues and homoeologues (chromosomes $56,38,37,11$ and chromosomes 33, 21, 9, 1) which are associated through a short nonhomologously paired segment and joined nonhomologously to chromosome 26 by a short synaptonemal complex. Finally the association between chromosomes $42,2,12,19$ and 4 may represent a pentavalent or more likely a quadrivalent where chromosome 12 is nonhomologously paired with chromosome 4.

As in early and mid zygotene nuclei of euploid wheat (16), the reconstructed nucleus contained regions of intimately associated and entangled chromosome segments, knots, through which the continuity of the lateral components and synaptonemal complexes could not be identified. These knots represent the major obstacle for tracing the chromosomes from telomere to telomere. The reconstructed nucleus contained six knots (Figure 10). The area of the boxes in Figure 10 is proportional to the number of lateral components entering the knot, the number of lateral components entering each of the six knots 
B. Wischmann: Chromosome pairing in wheat
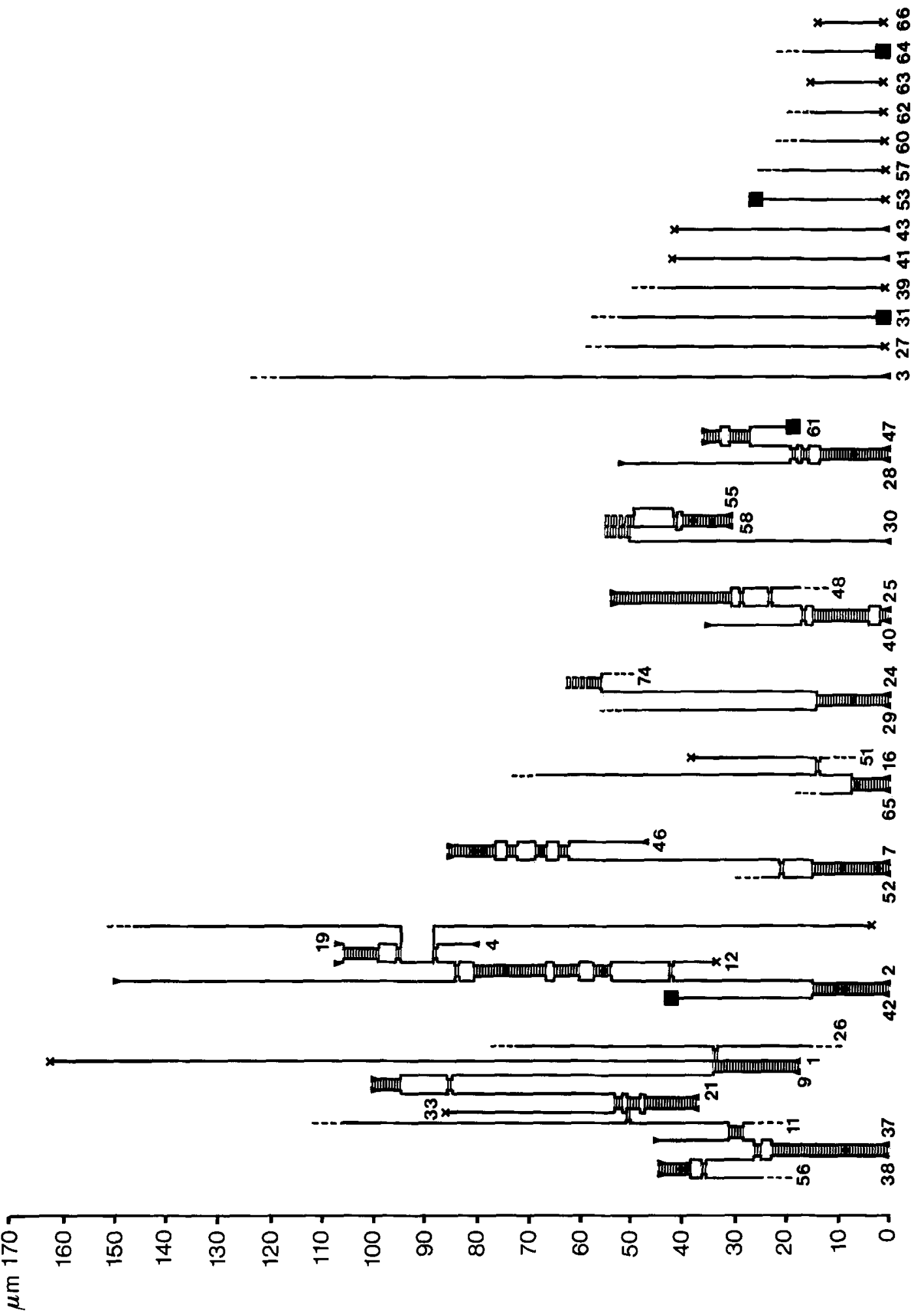
B. WISCHMANN: Chromosome pairing in wheat

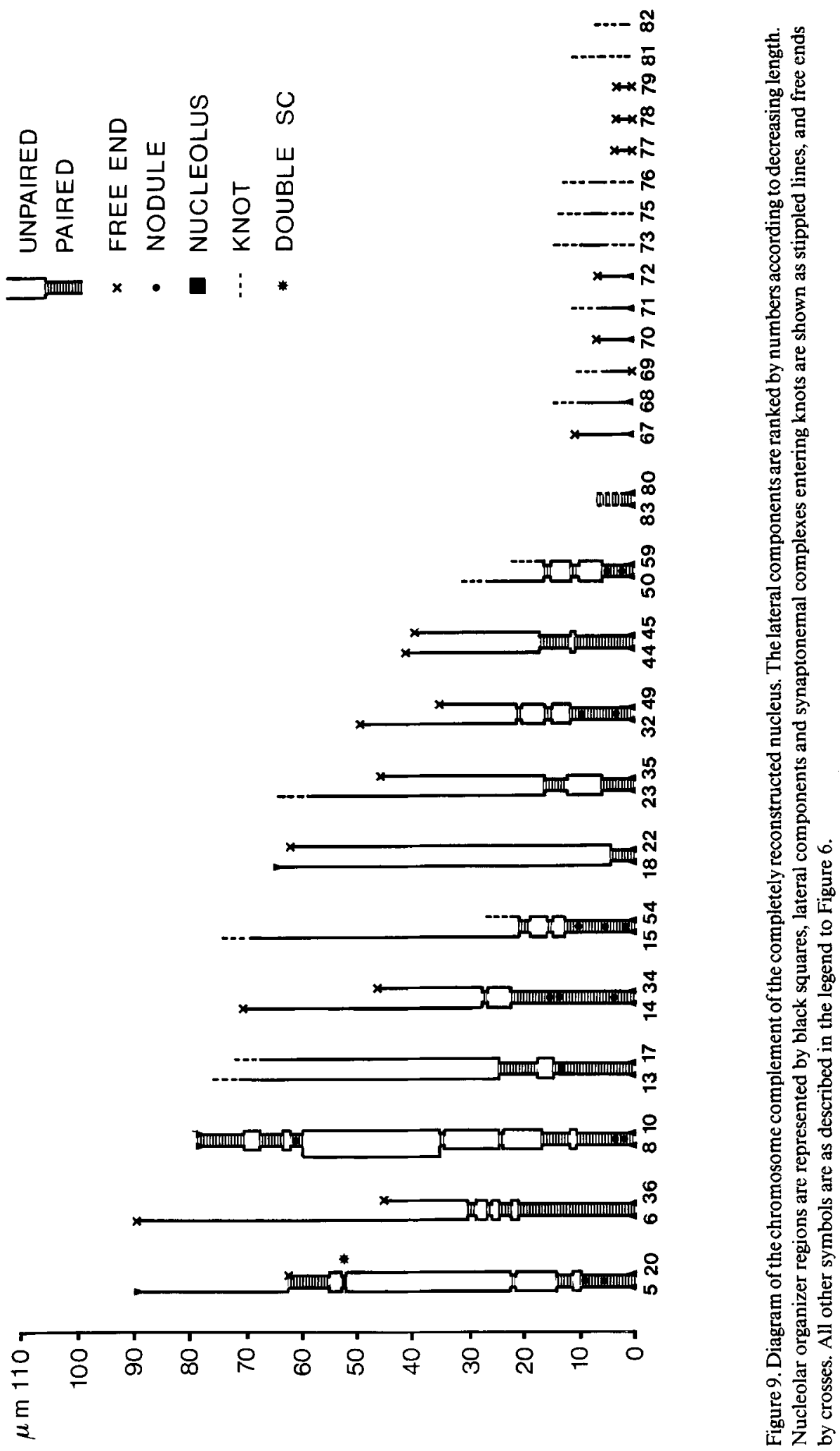

Carlsberg Res. Commun. Vol. 51, p. 1-25, 1986 
B. WISCHMANN: Chromosome pairing in wheat

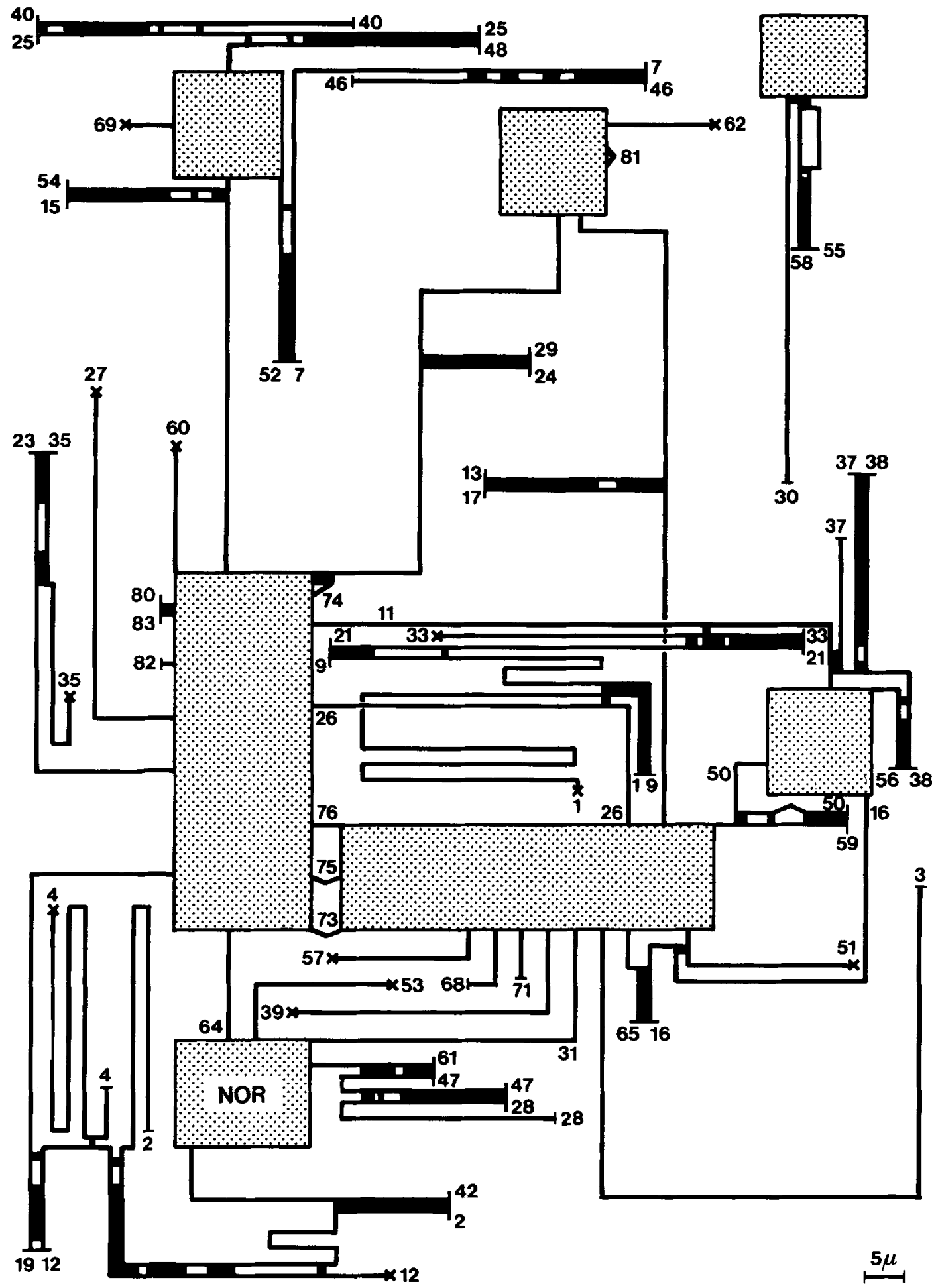


Figure 10. A diagrammatic representation of the synaptonemal complexes (thick lines) and unpaired lateral components (thin lines) which enter knots (hatched boxes) and/or the nucleolus organizing region (NOR). The size of the knots and the NOR are proportional to the number of the entering lateral components. Telomeres attached to the nuclear envelope are represented by bars and free ends by crosses. The numbers relate to the length of the lateral components as explained in the legend to Figure 9.

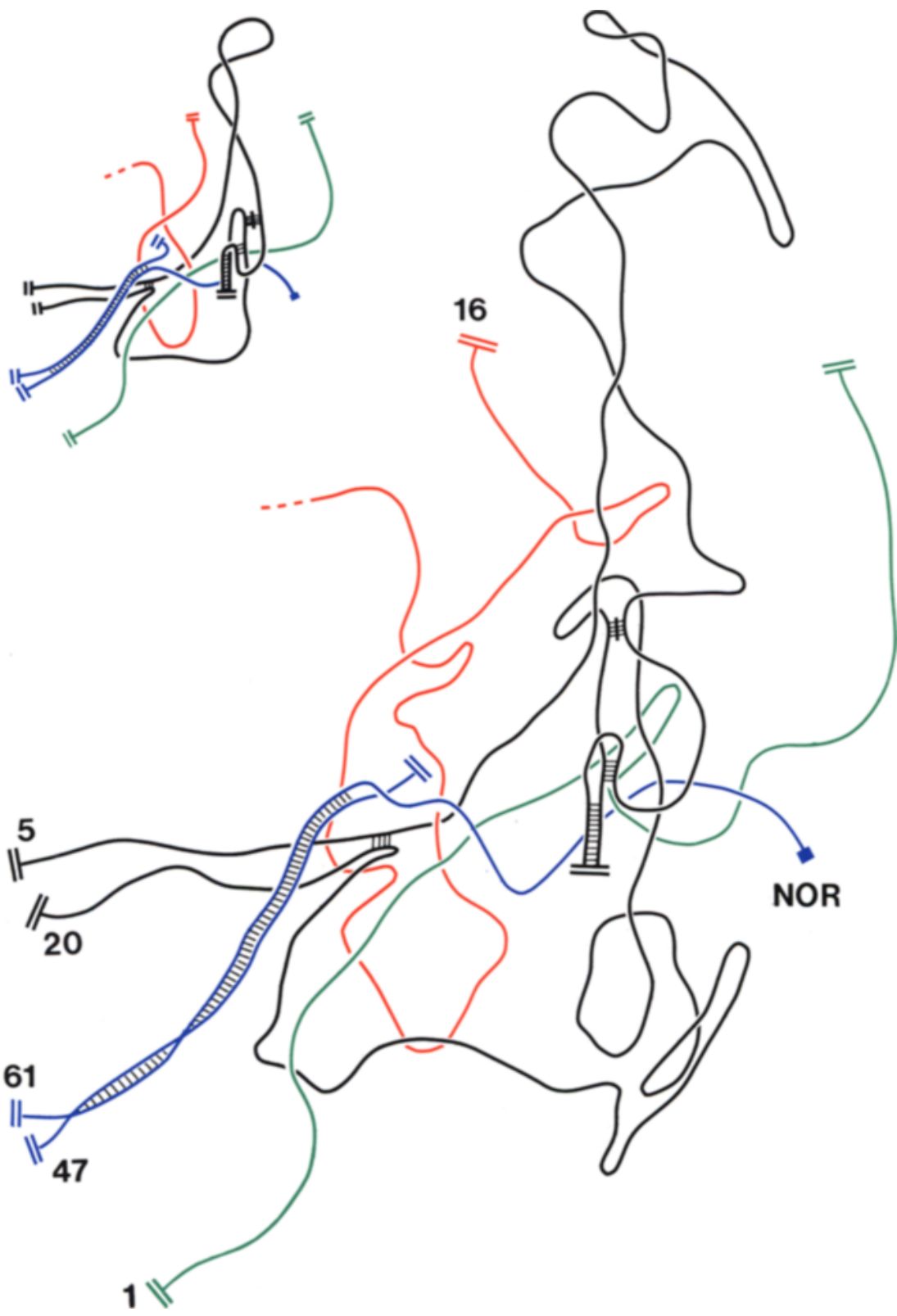

Figure 11. A reconstruction of the partially paired lateral components 5 and 20 which interlock lateral components 61 (blue), 1 (green) and 16 (red). Chromosome segments are delimited by two parallel lines. The single lines are stippled where the lateral components enter a knot. A more schematic representation of the interlocking event is shown as an insert. 

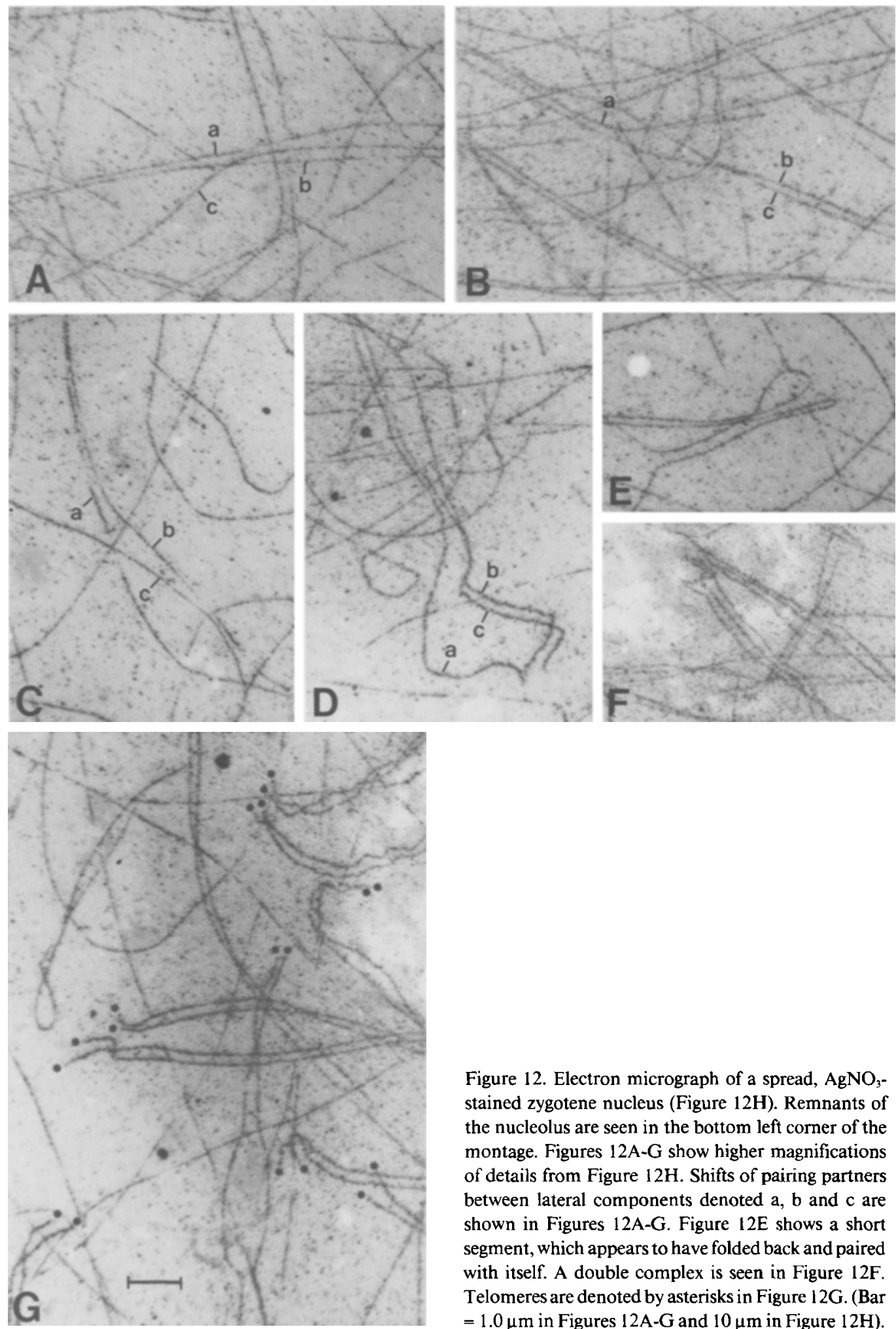

Figure 12. Electron micrograph of a spread, $\mathrm{AgNO}_{3}$ stained zygotene nucleus (Figure 12H). Remnants of the nucleolus are seen in the bottom left corner of the montage. Figures 12A-G show higher magnifications of details from Figure $12 \mathrm{H}$. Shifts of pairing partners between lateral components denoted $a, b$ and $c$ are shown in Figures 12A-G. Figure 12E shows a short segment, which appears to have folded back and paired with itself. A double complex is seen in Figure $12 \mathrm{~F}$. Telomeres are denoted by asterisks in Figure 12G. (Bar $=1.0 \mu \mathrm{m}$ in Figures $12 \mathrm{~A}-\mathrm{G}$ and $10 \mu \mathrm{m}$ in Figure $12 \mathrm{H}$ ). 
B. WISChMANN: Chromosome pairing in wheat

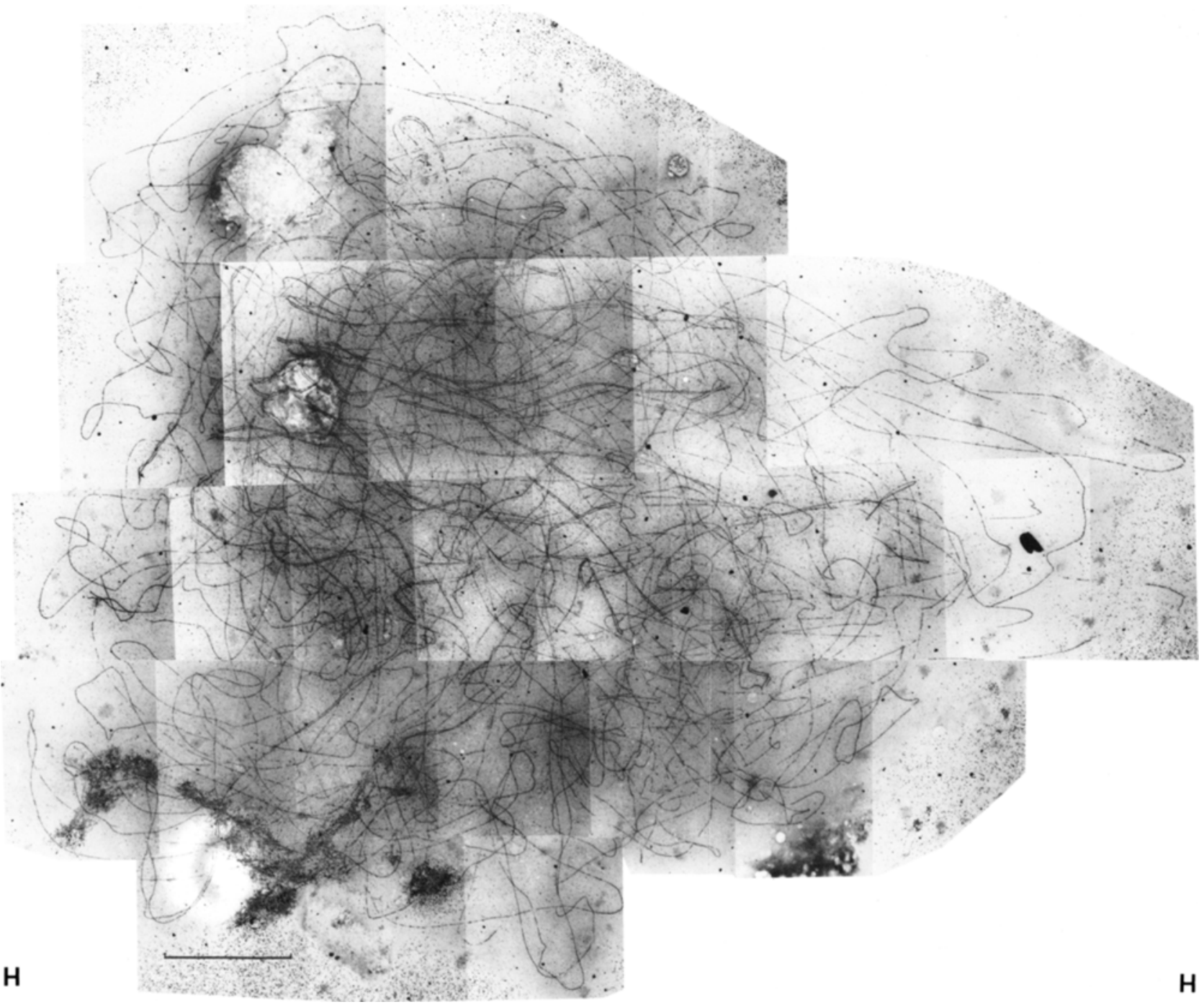


Table 1. Length of lateral components (LC) and synaptonemal complexes (SC) and degree of pairing in five spread zygotene nuclei. s. d., standard deviation.

\begin{tabular}{llll}
\hline $\begin{array}{l}\text { Nucleus } \\
\text { number }\end{array}$ & $\begin{array}{l}\text { LC length } \\
\mu \mathrm{m}\end{array}$ & $\begin{array}{l}\text { SC length } \\
\mu \mathrm{m}\end{array}$ & $\begin{array}{l}\text { Pairing } \\
\%\end{array}$ \\
\hline 1 & 3262 & 131 & 8 \\
2 & 4850 & 421 & 17 \\
3 & 4682 & 726 & 31 \\
4 & 4008 & 600 & 30 \\
5 & 3928 & 730 & 37 \\
\hline Mean \pm s.d. & $4146 \pm 639$ & $522 \pm 252$ & $25 \pm 12$ \\
\hline
\end{tabular}

being $16,14,5,4,4$ and 3 . The lateral components of the individual pairing associations often entered different knots. The knots consisted almost exclusively of unpaired chromosomes and only in two cases did a synaptonemal complex and in one case a short segment of double synaptonemal complex enter these regions (Figure 10).

One interlocking was revealed in the reconstruction of the complement. As shown in Figure 11, the partly paired bivalent (chromosome numbers 5 and 20) interlocks three unpaired lateral components (chromosome numbers 16, 1 and 61).

Two double synaptonemal complexes (Figure 8 ) and several small polycomplexes were seen either free in the nucleoplasm or associated with one or more unpaired lateral components.

A total of 33 recombination nodules was identified (Figures 4B and 9). These were indistinguishable in structure and dimensions from those described in normal wheat $(12,16)$. In only one of the presumptive trivalents (chromosome $52,7,46$, Figure 9) were recombination nodules present in the paired segment on both sides of the site of pairing partner exchange.

\subsection{Spreading of meiotic nuclei}

\subsubsection{General aspects}

Chromosome complements from spikes of 12 different plants triisosomic for the long arm of chromosome 5B were analyzed after spreading. Several hundred spread and silver stained nuclei were examined under the light microscope and of the large number of nuclei also analyzed in the electron microscope, 27 nuclei from five different plants were photographed for a more detailed analysis.

In spread and sectioned nuclei of normal wheat the pachytene stage is characterized by complete or almost complete pairing of the lateral components into synaptonemal complexes. This is accompanied by a change of the attachment sites of the telomeres on the nuclear envelope from the localized zygotene arrangement to a more uniform distribution at pachytene (12, HoLM, personal communication). The shape of the spread nuclei is stage dependent, zygotene nuclei being round while pachytene nuclei are irregularly elongated. The several hundred spread nuclei from plants triisosomic for chromosome 5BL only included zygotene nuclei with stretches of synaptonemal complexes and long continuous stretches of lateral components and diplotene nuclei in which the lateral components were more or less degraded and the remnants of the synaptonemal complexes were present as discrete fragments. Pachytene nuclei as defined by the criteria described above were never observed in the triisosomic 5BL plants analysed. Of the 27 photographed nuclei 15 were in zygotene and 12 in diplotene.

\subsubsection{Zygotene}

The fine structure of a spread zygotene nucleus is shown in Figure $12 \mathrm{H}$. As in other zygotene nuclei the terminal paired segments showed a polarized distribution resulting from the aggregation of telomeric attachment sites on the nuclear envelope (Figure 12G). The central 


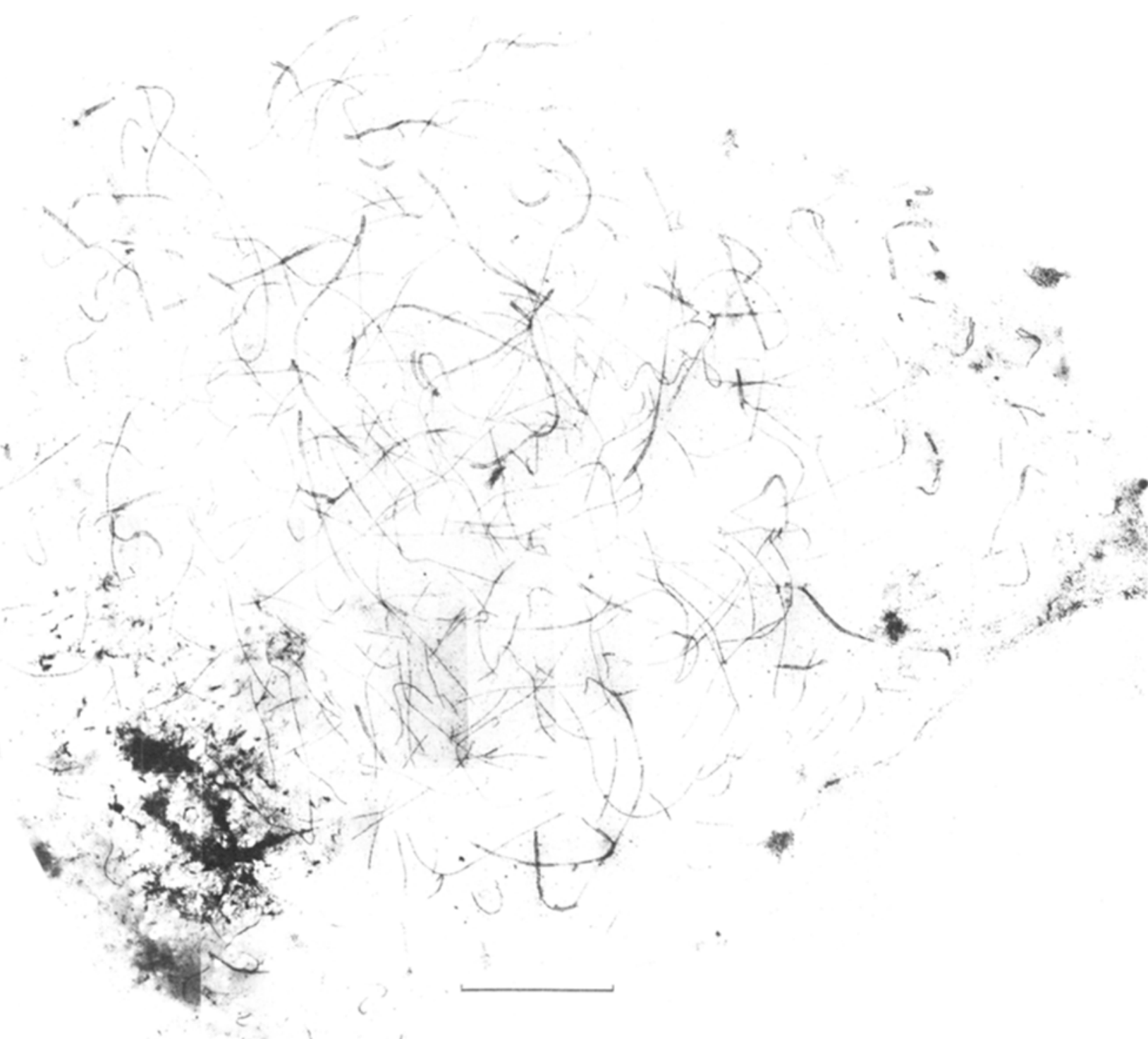

Figure 13. Electron micrograph of a spread, $\mathrm{AgNO}_{3}$-stained nucleus at early-mid diplotene. The dissolution of the synaptonemal complexes and the unpaired lateral components has begun. $(\mathrm{Bar}=10 \mu \mathrm{m})$. 

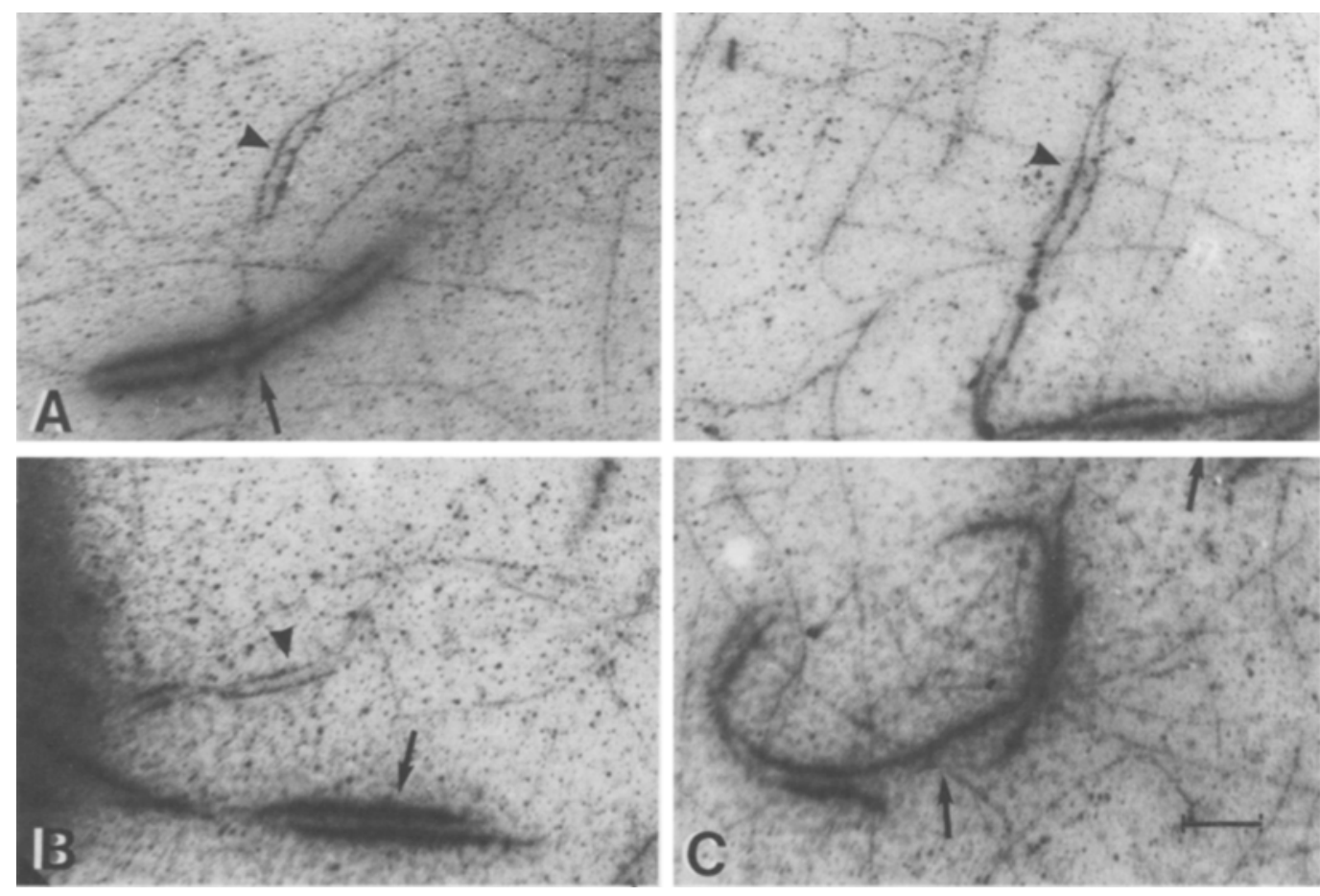

Figure 14. Electron micrograph of a spread, $\mathrm{AgNO}_{3}$-stained nucleus at late diplotene (Figure 14D). In Figures $14 \mathrm{~A}-\mathrm{C}$ are shown higher magnifications of synaptonemal complex segments with thickened lateral components (arrow) and segments with unmodified lateral components (arrowheads). (Bar $=1 \mu \mathrm{m}$ in Figures $14 \mathrm{~A}-\mathrm{C}$ and 10 $\mu \mathrm{m}$ in Figure 14D).

component of the synaptonemal complex is normally not apparent, but paired segments can easily be recognized as parallel stretches of lateral component segments. The lateral components were frequently interrupted and a complete tracing of the 43 chromosomes could not be performed in any of the spread nuclei. Some of the discontinuities are probably introduced mechanically during the flattening of the nuclei as they are air dried. It is, however, likely that some of the discontinuities reflect an initiation of degradation of the lateral components, either due to a liberation of proteolytic enzymes during maceration and spreading or due to the beginning of the diplotene phase of synaptonemal complex degradation. Often the interruptions of the lateral components occurred with some periodicity.

Knots of entangled lateral components as seen in the reconstructed nucleus, were not visible in the spreadings. It is thus likely that the knots open up and become undetectable during the spreading procedure which causes the nuclei to swell and the chromatin to disperse.

The length of the synaptonemal complex and the total length of the lateral components were measured in five of the zygotene nuclei and the percentage of pairing was calculated (Table I). One of the nuclei (number one) was significantly less paired than the other four nuclei $(8 \%$ versus $17-37 \%)$. In this nucleus the complement length was somewhat shorter $(3,262 \mu \mathrm{m})$ than in nuclei with a higher degree of pairing $(3,928 \mu \mathrm{m}-4,850$ $\mu \mathrm{m})$.

Shifts of pairing partners were frequently found. For example nucleus number 3 (Figure $12 \mathrm{H})$ contained one complex association involving thirteen lateral component segments. In addition, this nucleus contained one association of six, one of five, two of four and three associ- 


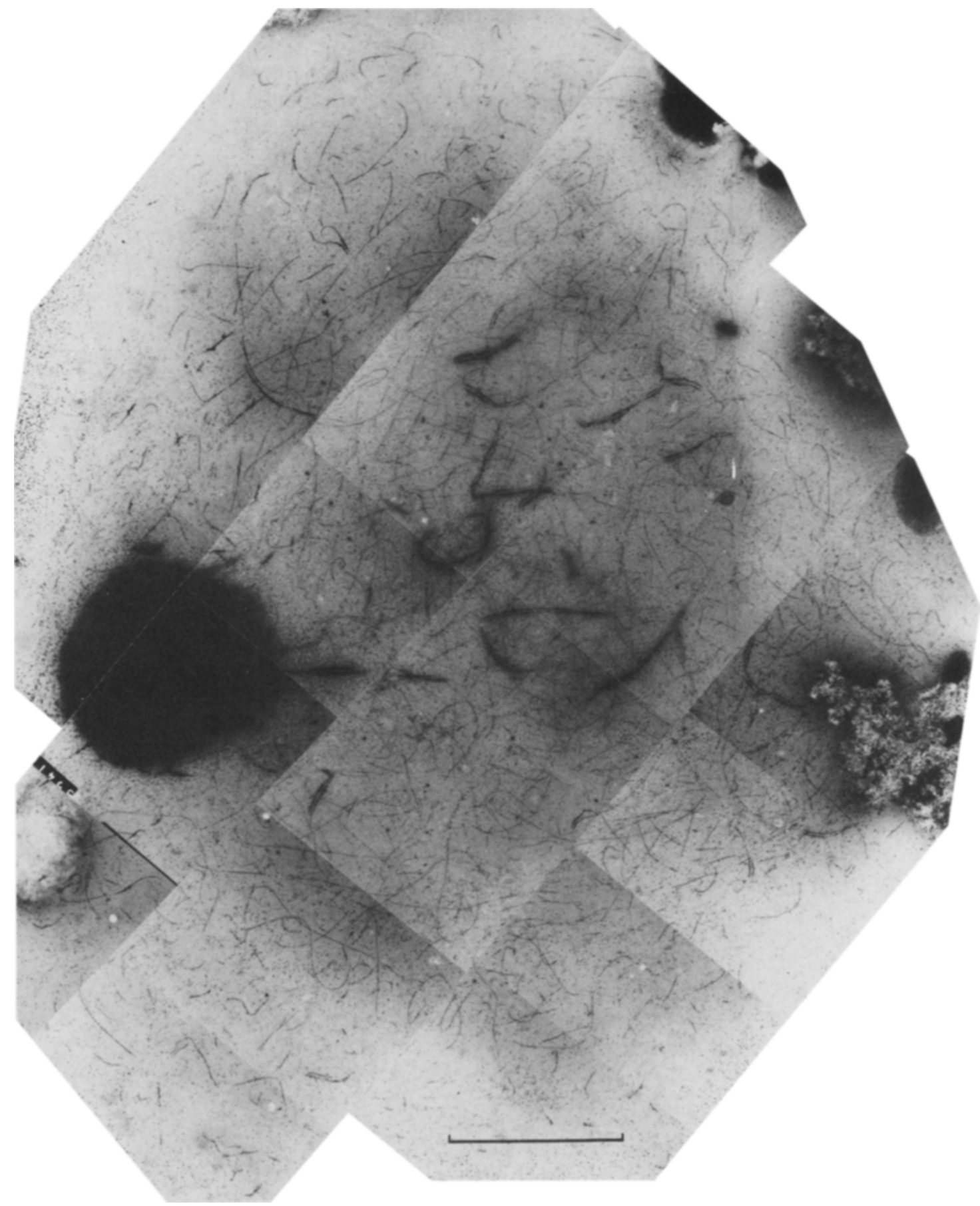


Table II. Length of lateral components (LC) and synaptonemal complexes (SC) in spread nuclei at early-mid diplotene. s. d., standard deviation.

\begin{tabular}{lll}
$\begin{array}{l}\text { Nucleus } \\
\text { number }\end{array}$ & $\begin{array}{l}\text { LC length } \\
\mu \mathrm{m}\end{array}$ & $\begin{array}{l}\text { SC length } \\
\mu \mathrm{m}\end{array}$ \\
\hline 6 & 2559 & 277 \\
7 & 2172 & 348 \\
8 & 2403 & 33 \\
\hline Mean \pm s. d. & $2378 \pm 195$ & \\
\hline
\end{tabular}

ations involving three lateral components (Figure 12F). Figure 12 A-D shows some of the pairing partner shifts. Figure 12E shows a foldback pairing, most likely between nonhomologous chromosome segments.

\subsubsection{Diplotene}

The twelve photographed diplotene nuclei were divided into an early-mid diplotene stage (6 nuclei) and a late diplotene stage (6 nuclei). The first stage was characterized by a large variation in the length of the synaptonemal complex segments and by the presence of many long stretches of lateral components (Figure 13) while at late diplotene the synaptonemal complex segments were more uniform in length and the lateral components were extensively degraded (Figure 14D). At diakinesis remnants of the synaptonemal complexes and the lateral components could no longer be recognized.

The length of the lateral components and the synaptonemal complexes was measured in three early-mid (Table II) and six late diplotene nuclei (Table III). At late diplotene the mean combined length of the synaptonemal complexes amounted to $83 \pm 39 \mu \mathrm{m}$. The difference in length between the nuclei was substantial, probably resulting from initial differences in the amount of pairing and/or reflecting different steps in the degradation of the complex. Similar differences were observed in early-mid diplotene, where particularly nucleus number 8 had a very short synaptonemal complex length (33 $\mu \mathrm{m})$.

In some nuclei the retained synaptonemal complex segments were confined to a smaller
Table III. Length of the lateral components (LC) and synaptonemal complexes (SC) in spread nuclei at late diplotene. s. d., standard deviation.

\begin{tabular}{lll}
$\begin{array}{ll}\text { Nucleus } \\
\text { number }\end{array}$ & $\begin{array}{l}\text { LC length } \\
\mu \mathrm{m}\end{array}$ & $\begin{array}{l}\text { SC length } \\
\mu \mathrm{m}\end{array}$ \\
\hline 9 & 2891 & 138 \\
10 & 1956 & 118 \\
11 & 1413 & 44 \\
12 & 1342 & 43 \\
13 & 1108 & 71 \\
14 & 1067 & 85 \\
\hline Mean \pm s. d. & $1630 \pm 695$ & $83 \pm 39$ \\
\hline
\end{tabular}

part of the flattened nucleus (Figure 14D) while in others they were more uniformly distributed (Figure 13). The polarized distribution shows that the aggregation of the telomeres typical of zygotene nuclei has been retained while the more uniform distribution may indicate some movement of the distally paired segments after zygotene.

In five of the six late diplotene nuclei two distinctly different types of synaptonemal complex segments were observed, one with thickened lateral components and one with a normal appearance of lateral components (Figures 14AC). The two types were about equally frequent ( 20 versus 22 , Table IV), the mean total number of segments being 41 . The last nucleus contained segments which all appeared normal.

\section{DISCUSSION}

\subsection{Chromosome pairing}

The present light and electron microscopical analyses of chromosome pairing in wheat plants triisosomic for the long arm of chromosome 5B have shown the following. 1) In contrast to the situation in normal euploid wheat where chromosome pairing reaches completion or almost so, pairing is arrested in triisosomic $5 \mathrm{~B}$ plants at an early stage of zygotene (average of $25 \%$ pairing). 2) A large number of associations of more than two chromosomes are formed due to chromosome pairing and synaptonemal complex formation between chromosomes which are not homologous. Although the quantitative 
Table IV. Number of modified (MSC) and unmodified (USC) synaptonemal complex segments at late diplotene. S.d., standard deviation.

\begin{tabular}{llll}
\hline $\begin{array}{l}\text { Nucleus } \\
\text { number }\end{array}$ & $\begin{array}{l}\text { Number of MSC } \\
\text { segments }\end{array}$ & $\begin{array}{l}\text { Number of USC } \\
\text { segments }\end{array}$ & $\begin{array}{l}\text { Total number } \\
\text { of SC segments }\end{array}$ \\
\hline 9 & 26 & 29 & 55 \\
10 & -20 & $-{ }^{-2}$ & 33 \\
11 & 19 & 10 & 50 \\
12 & 20 & 32 & 29 \\
13 & 17 & 11 & 52 \\
14 & $20 \pm 3$ & $22 \pm 11$ & 28 \\
\hline Mean \pm s.d. & & $41 \pm 12$ \\
\hline
\end{tabular}

a, all segments with unmodified lateral components

observations on their frequency are limited in this study, it is apparent that such multiple associations form at a high frequency.

The differences between euploid and triisosomic 58L wheat are, however, not correlated with other characteristics of the nuclei. The structure and dimensions of the synaptonemal complexes are indistinguishable from that reported in normal wheat and the total complement length in the reconstructed nucleus $(3,443 \mu \mathrm{m})$ and the spread nuclei (mean of $4,146 \mu \mathrm{m}$ ) are very similar to that reported for a reconstructed $(3,784 \mu \mathrm{m}, 12)$ zygotene nucleus of euploid wheat. The initiation and early progression of pairing also appear normal with an aggregation of the attachment sites for the telomeres and a preferential initiation of synaptonemal complex formation in the telomeric segments. Interstitial initiation of chromosome pairing and synaptonemal complex formation also appears to proceed as in normal wheat, although being considerably less frequent.

As discussed in section 3.3.2, associations involving two chromosomes probably represent partially paired homologous chromosomes, while the associations of three to six chromosomes most likely consist of homologues and homoeologues. Such associations may then join into more complex configurations by nonhomologous synapsis. This interpretation is supported by the following: 1) It is a reasonable assumption that partly homologous chromosomes pair more readily than nonhomologous chromosomes. 2)
Multivalents, in particular trivalents, and heteromorphic bivalents are seen at metaphase I in these plants $(10$, and this study) indicating crossing over and chiasma formation between homoeologues.

It is, however, evident that not all quadrivalents and trivalents observed at zygotene are maintained until metaphase I. FELDMAN (10) reported that trivalents were present at a frequency of 0.41 per cell, that quadrivalents and pentavalents were rare while heteromorphic bivalents were rather frequent. Most of the multiple associations are thus corrected during zygotene to configurations involving fewer chromosomes or (as discussed in section 4.2.) fall apart during the diplotene-metaphase I interval due to lack of crossing over and chiasma formation between some of the involved chromosomes.

Only a single interlocking event was directly traceable in the reconstructed nucleus. Since only few chromosomes could be traced from telomere to telomere this number is not representative of the actual frequency of interlocking. The entangled chromosome segments comprising the knots may for example arise partially as a consequence of interlocking (see 16).

\subsection{Crossing over and chiasma formation}

The fully reconstructed nucleus which had $25 \%$ of its complement paired with a synaptonemal complex contained 33 recombination 
nodules which were indistinguishable in structure and dimensions from those described from normal wheat $(12,16)$. НовоLTH (12) counted 97 nodules in a nearly fully paired late zygotene nucleus and 88 nodules in a pachytene nucleus of normal wheat. On the asumption that the unpaired segments in the present early zygotene nucleus upon pairing have the same probability for acquiring nodules as those segments already paired, 132 nodules are expected. Since distal segments generally acquire more nodules than interstitial and proximal segments (14), the number of nodules per unit length of synaptonemal complex may not be different in the two genotypes.

Assuming that all the observed nodules have performed a crossover the following stable chromosome configurations can be predicted: Three trivalents (from the association of nine chromosomes, the association of five chromosomes and the association of three chromosomes where nodules were present on both sides of the region of pairing partner exchange), several homomorphic and heteromorphic bivalents and a number of univalents. As outlined in section 4.1 FELDMAN (10) observed a mean of only 0.41 trivalents per nucleus and very few multivalents which implies either lack of crossing over between the chromosomes or transformation of the multiple associations into bivalents and/or univalents before crossing over occurred.

The orderly degradation of the synaptonemal complex and the unpaired lateral components during diplotene is revealed with remarkable clarity in the spread nuclei. A large number of analyses from serially sectioned diplotene nuclei from a variety of organisms $(13,15,24,25,26$, 29 ) have shown that segments of synaptonemal complexes are retained at sites where crossing over has occurred and thus represent the early chiasmata. These segments are eliminated at the diplotene/diakinesis transition when formation of a more elaborate chromatin chiasma has taken place.

Investigations of serially sectioned diplotene nuclei of human spermatocytes showed that degradation of the synaptonemal complex was initiated at a low number of sites, generating a population of synaptonemal complex segments which in number and distribution correlated with the number and distribution of crossovers as deduced from the analysis of recombination nodules. At mid-late diplotene the number and distribution of distinct synaptonemal complex segments was the same as at early diplotene, showing that degradation at this stage only occurred from the ends of the segments present at early diplotene. Partially degraded segments were often seen flanking the well defined synaptonemal complex segments (15).

The present study revealed a mean of 41 retained synaptonemal complex segments at late diplotene, of which half possessed thickened lateral components, and the other half had lateral components of normal appearance. It is conceivable that only segments with modified lateral components contain a crossover, while the normal appearing segments may be in the process of elimination. The virtual identity between the number of segments with modified lateral components ( 20 segments) and the number of chiasmata as seen at metaphase I in the light microscope, namely 18 chiasmata in this study and 20 chiasmata in (10) would support this hypothesis. It is, however, unlikely that the number and distribution of chiasmata at metaphase I is identical to the number and distribution of crossovers initially formed at pachytene. At metaphase I most chiasmata are terminal and a substantial reduction in chiasma frequency and a change in their distribution might have occurred before metaphase $I$ as has indeed been reported for human spermatocytes (15). Hence it is conceivable that all retained synaptonemal complex segments in the present material are associated with crossovers.

\subsection{Dosage effect of the long arm of chromosome 5B}

The present ultrastructural investigations have shown that the presence of three isochromosomes for the long arm of chromosome 5B causes extensive asynapsis, retainment of chromosome and bivalent interlockings up to metaphase I, and pairing and crossing over between supposedly homoeologous chromosomes. This is in agreement with the light microscopic observations by FELDMAN (10). These effects cannot be attributed alone to the physical presence of the three isochromosomes, since chromosome 
pairing in lines triisosomic for the long arm of chromosome 5D appears to be complete and mostly bivalents are seen at later stages (10).

According to FELDMAN (10) the high frequency of interlocking at metaphase $I$ as well as the asynapsis in triisosomic 5BL plants reflect a suppression of an ordered premeiotic chromosome arrangement causing a random distribution of chromosomes in the premeiotic nuclei due to six doses of the $\mathrm{Ph}$ gene. An alternative and more likely explanation is that the high frequency of interlocking at metaphase I results from the incomplete synapsis. It is apparent from a number of investigations that interlockings first are resolved when the interlocked segments no longer can move freely, i.e., if pairing is nearly completed or if the interlocked and interlocking chromosomes move in different directions. Hence, a resolution of all interlockings by breakage and reunion may only occur if pairing is completed, i.e., the retention of interlockings up to metaphase I may be regarded as a qualitative and maybe also a quantitative measure of the degree of pairing completion.

YACOBI et al. (28) counted the number of interlockings at metaphase $I$ in a large number of lines with different doses of the long arm of chromosome 5B.A linearcorrelation was observed when comparing the number of interlockings per ring bivalent with the number of copies of $5 \mathrm{BL}$, the frequency ranging from 0.002 interlockings per ring bivalent in euploid wheat to 0.135 in triisosomic $5 \mathrm{BL}$ plants. This correlation may well reflect reduced chromosome pairing due to the increasing copy number of 5BL. In agreement with this idea preliminary observations of chromosome pairing and synaptonemal complex formation in wheat diisosomic for the long arm of chromosome $5 \mathrm{~B}$ have shown an arrest of pairing at a somewhat more advanced stage of pairing than in triisosomic 5BL plants (HOLM, personal communication).

YACOBI et al. (28) also noted a reduction in interlocking frequency in genotypes where the short arm of chromosome $5 \mathrm{~B}$ was present and concluded that the short arm of chromosome 5B contained a gene which counteracted the effect of the Ph gene. It is thus conceivable that genes on this arm are important for the chromosome pairing. The effect may, however, be rather low since the number of interlockings at metaphase I in lines mono- and ditelosomic for the long arm of chromosome 5B is low (0.20 and 0.14 interlockings per cell versus 0.04 interlockings per cell in euploid wheat). That genes on the short arm have some effect on meiosis can also be inferred from the observations by BENNETT et al. (5) who observed an increased duration of meiosis in genotypes lacking this chromosome arm.

A number of investigations have drawn attention to a resemblance between asynapsis induced by the administration of colchicine at an early stage of pairing $(7,8)$, asynapsis induced by growing diisosomic $5 \mathrm{BL}$ plants at $15^{\circ} \mathrm{C}(10,21)$ and the asynapsis observed in triisosomic $5 \mathrm{BL}$ plants. In all three situations several univalents are present and the chiasma frequencies are rather similar.

Neither colchicine nor low temperature do, however, appear to induce multivalent formation in normal wheat and diisosomic 5BL plants to the same extent as the presence of six doses of $5 \mathrm{BL}$. This indicates that the presence of six copies of the long arm of chromosome 5B exerts some effect on pairing and recombination in addition to partially arresting synapsis. It is at present not known whether colchicine or low temperature also leads to multivalent formation at zygotene implying that only crossing over is differentially affected. Preliminary observations from diisosomic 5BL plants suggest that the number of multiple associations at zygotene in these plants is much lower than in triisosomic 5BL plants (HOLM, personal communication). Hence an increase in copy number of $5 B L$ from four to six doses seems to cause a further reduction in the extent of pairing and to permit pairing and recombination also between homoeologues.

\section{ACKNOWLEDGEMENTS}

I would like to express my gratitude to Professor DITER VON WETTSTEIN for reviewing this manuscript. I am indebted to Dr. Preben B. HOLM and Dr. SøREN W.RASMUSSEN for practical guidance and encouragement throughout this work. MSc. XINGZHI WANG is thanked for 
expert advice concerning N-banding. The outstanding technical assistance of BøRGE PETERSEN, JEAN SAGE, and BIBI STAMPE ANDERSEN is also gratefully acknowledged. This work was financially supported by grants BIO-E-417DK-G and BI6-168-DK from the Commission of the European Communities to D. vON WETTSTEIN.

\section{REFERENCES}

1. ANDERSON, P. J.: Purification and quantitation of glutaraldehyde and its effect on several enzyme activities in skeletal muscle. J. Histochem. Cytochem. 15, 652-661 (1967)

2. Appels, R., W.L.Gerlach, E.S.DenNis, H.SWIFT \& W. J. PEACOCK: Molecular and chromosomal organization of DNA sequences coding for the ribosome RNAs in cereals. Chromosoma 78, 293311 (1980)

3. Avivi, L., M. Feldman \& M. Brown: An ordered arrangement of chromosomes in the somatic nucleus of common wheat, Triticum aestivum $L$. I. Spatial relationships between chromosomes of the same genome. Chromosoma 86, 1-16 (1982)

4. Avivi, L., M. Feldman \& M. Brown: An ordered arrangement of chromosomes in the somatic nucleus of common wheat, Triticum aestivum L. II. Spatial relationships between chromosomes of different genomes. Chromosoma 86, 17-26(1982)

5. BennetT, M. D., G. A. Dover \& R. Riley: Meiotic duration in wheat genotypes with or without homoeologous meiotic chromosome pairing. Proc. R. Soc. Lond. B. 187, 191-207 (1974)

6. Bloom, S. E. \& C. Goodpasture: An improved technique for selective silver staining of nucleolar organizer region in human chromosomes. Hum. Genet. 34, 199-206 (1976)

7. Dover, G. A. \& R. RILEY: The effect of spindle inhibitors applied before meiosis on meiotic chromosome pairing. J. Cell Sci. 12, 143-161 (1973)

8. Driscoll, C. J., N. L. Darvey \& H. N. Barber: Effect of colchicine on meiosis in hexaploid wheat. Nature 216, 687-688 (1967)

9. ENDO, T. R. \& B. S. Gill: Somatic karyotype, heterochromatin distribution, and the nature of chromosome differentiation in common wheat, Triticum aestivum L. em Thell. Chromosoma 89, 361-369 (1984)

10. Feldman, M.: The effect of chromosomes 5B, 5D and $5 A$ on chromosomal pairing in Triticum aestivum. Proc. Nat. Acad. Sci. USA 55, 14471453 (1966)
11. GoOdPasture, C. \& S. E. BloOM: Visualization of nucleolar organizer regions in mammalian chromosomes using silver staining. Chromosoma 53, 37-50 (1975)

12. HobOlth, P.: Chromosome pairing in allohexaploid wheat var. Chinese Spring. Transformation of multivalents into bivalents, a mechanism for exclusively bivalent formation. Carlsberg Res. Commun. 46, 129-173 (1981)

13. Holm, P. B. \& S. W. Rasmussen: Chromosome pairing, recombination nodules and chiasma formation in diploid Bombyx males. Carlsberg Res. Commun. 45, 483-548 (1980)

14. Holm, P. B. \& S. W. Rasmussen: Human meiosis VI. Crossing over in human spermatocytes. Carlsberg Res. Commun. 48, 385-413 (1983)

15. Holm. P. B. \& S. W. Rasmussen: Human meiosis VII. Chiasma formation in human spermatocytes. Carlsberg Res. Commun. 48, 415-456 (1983)

16. Jenkins, G.: Chromosome pairing in Triticum aestivum cv. Chinese Spring. Carlsberg Res. Commun. 48, 255-283 (1983)

17. JeNKINS, G.: Synaptonemal complex formation in hybrids of Lolium temulentum x Lolium perenne (L.). II. Triploid. Chromosoma 92, 387-390(1985)

18. JENKINS, G.: Synaptonemal complex formation in hybrids of Lolium temulentum $x$ Lolium perenne (L. ). III. Tetraploid. Chromosoma (in press)

19. Rasmussen, S. W. \& P. B. Holm: Human meiosis II. Chromosome pairing and recombination nodules in human spermatocytes. Carlsberg Res. Commun. 43, 275-327 (1978)

20. Rasmussen, S. W. \& P. B. Holm: Chromosome pairing in autotetraploid Bombyx females. Mechanism for exclusive bivalent formation. Carlsberg Res. Commun. 44, 101-125 (1979)

21. Riley, R. \& V. ChapMaN: Effect of 5BS in suppressing the expression of altered dosage of $5 \mathrm{BL}$ on meiotic chromosome pairing in Triticum aestivum. Nature 216, 60-62 (1967)

22. Riley, R. \& C. KempanNa: The homoeologous nature of the non-homologous meiotic pairing in Triticum aestivum deficient for chromosome $\mathrm{V}$ (5B). Heredity 18, 287-306 (1963)

23. SEARS, E. R.: Genetic control of chromosome pairing in wheat. Ann. Rev. Genet. 10, 31-51 (1976)

24. SolarI, A. J.: The behavior of chromosomal axes during diplotene in mouse spermatocytes. Chromosoma 31, 217-230 (1970)

25. WestergaARd, M. \& D. von Wettstein: The meiotic cycle in an ascomycete. In effects of radiation on meiotic systems. Internat. Atom. Energy Agency. Panel Proc. Sci.,Pub. 173, 113-121 (1968)

26. WestergaARD, M. \& D. von Wettstein: Studies 
on the mechanism of crossing-over IV. The molecular organization of the synaptonemal complex in Neottiella (Cooke) Saccardo (Ascomycetes). Compt. Rend. Trav, Lab. Carlsberg 37, 239-268 (1970)

27. Wettstein, D. von, S. W. Rasmussen \& P. B. Holm: The synaptonemal complex in genetic segregation. Ann. Rev. Genet. 18, 331-431 (1984)
28. Yacobi, Y. Z., T. Mello-Sampayo \& M. FeldMAN: Genetic induction of bivalent interlocking in common wheat. Chromosoma 87, 165-175 (1982)

29. ZICKLER, D.: Development of the synaptonemal complex and the "recombination nodules" during meiotic prophase in the seven bivalents of the fungus Sordaria macrospora Auersw. Chromosoma 61, 289-316(1977)

Accepted by E. LuND 